\title{
Outer Boundaries of Self-Similar Tiles
}

\author{
Shawn Drenning, Judith Palagallo, Thomas Price, and Robert S. Strichartz
}

\section{CONTENTS}

1. Introduction

2. Boundaries of Components of the Interior

3. Examples

Acknowledgments

References
2000 AMS Subject Classification: Primary 52C20, 28A80

Keywords: Self-similar tile, outer boundary
There are many examples of self-similar tiles that are connected, but whose interior is disconnected. For such tiles we show that the boundary of a component of the interior may be decomposed into a finite union of pieces, each similar to a subset of the outer boundary of the tile. This is significant because the outer boundary typically has lower dimension than the full boundary. We describe a method to realize the outer boundary as the invariant set of a graph-directed iterated function system. The method works under a certain "finiteness" assumption. While it is not clear that this assumption always holds, and it is problematic to give a rigorous proof that it holds even in cases where it is "visually clear" that it holds, we give some examples where the method yields clear and nontrivial results. Details concerning the algorithms may be found at the website www.math.cornell.edu/ ${ }_{\text {sld32/Tiles.html. }}$

\section{INTRODUCTION}

We begin by discussing the simplest type of self-similar tile in the plane $\mathbb{R}^{2}$. Let $T$ denote the tile and $T_{i}=T+i$ denote its lattice translates, $i \in \mathbb{Z}^{2}$. We assume

$$
\mathbb{R}^{2}=\bigcup_{i \in \mathbb{Z}^{2}} T_{i}
$$

with disjoint interiors. We also assume there is a digit set $\mathcal{D} \subseteq \mathbb{Z}^{2}$ of cardinality $m^{2}$ so that

$$
\bigcup_{d \in \mathcal{D}} T_{d}=m T
$$

( $m$ denotes a positive integer, $m \geq 2$, and $m T$ is just the dilation of $T$ by the factor $m$ ). We assume that $\mathcal{D}$ is a complete set of residues for $\mathbb{Z}^{2} / m \mathbb{Z}^{2}$, and we may assume without loss of generality (just translate $T$ ) that $0 \in \mathcal{D}$. The self-similarity condition (1-2) implies that the whole tiling (1-1) is self-similar, since

$$
\bigcup_{d \in \mathcal{D}} T_{(d+m i)}=m T_{i}=m T+m i
$$

and

$$
\mathbb{R}^{2}=\bigcup_{i \in m \mathbb{Z}^{2}}(m T+i)
$$


is a tiling of the plane by $m \mathbb{Z}^{2}$ translates of $m T$. More generally, for every integer $\ell$ there exists a tiling, which we will call the level $\ell$ tiling,

$$
\mathbb{R}^{2}=\bigcup_{i \in m^{-\ell} \mathbb{Z}^{2}}\left(m^{-\ell} T+i\right)
$$

by $m^{-\ell} \mathbb{Z}^{2}$ translates of $m^{-\ell} T$. We will refer to the sets $\left(m^{-\ell} T+i\right)$ as tiles of level $\ell$, and denote the collection of these by $\mathcal{T}_{\ell}$.

Our main interest is the geometry of $T$. Many papers have already addressed this issue (see the references). The motivating question that we pose is the following: if $T$ is connected, but $\operatorname{int}(T)$ (the interior) is disconnected, what can be said about the connected components of $\operatorname{int}(T)$ ? In studying this question we were led to investigate the outer boundary of $T$. Let $B(T)$ denote the boundary of $T$. The outer boundary $b(T)$ (a subset of $B(T)$ ) is defined to be the boundary of the unbounded component of the complement of $T$. We will show that the geometry of $b(T)$ "contains" the geometry of the boundary of any component $C$ of $\operatorname{int}(T)$, in the sense that $B(C)$ can be written as a finite union of sets, each similar to a subset of $b(T)$. In particular, this implies that the dimension (in any sense) of $B(C)$ is bounded above by the dimension of $b(T)$. This is a significant estimate because in many cases $b(T)$ has a considerably smaller dimension than $B(T)$. We actually believe that the dimensions of $B(C)$ and $b(T)$ are equal and that the subsets of $b(T)$ needed to construct $B(C)$ may be described explicitly. We also believe that $b(T)$ may be decomposed into a finite collection of subsets that form the invariant set for a graph-directed iterated function system (IFS) with open set condition. This means in particular, that we can compute the dimension (box and Hausdorff are equal) of $b(T)$. We will examine in detail some examples where these expectations are satisfied.

Our approach might be described as "quasialgorithmic." We construct certain "algorithms," but at certain places these algorithms require human intervention. First, we describe an honest algorithm to approximate $T$. If we multiply Equation (1-2) by $m^{-1}$ and iterate we obtain

$$
T=\bigcup_{d \in \mathcal{D}_{\ell}}\left(m^{-\ell} T+d\right)
$$

where

$$
\mathcal{D}_{\ell}=\sum_{k=1}^{\ell} m^{-k} \mathcal{D}=\left\{\sum_{k=1}^{\ell} m^{-k} d_{k}: d_{k} \in \mathcal{D}\right\} .
$$

We interpret Equation (1-6) as a decomposition of $T$ into tiles of level $\ell$. Let $S$ denote the unit square. We take

$$
T^{(\ell)}=\bigcup_{d \in \mathcal{D}_{\ell}}\left(m^{-\ell} S+d\right)
$$

for our level $\ell$ approximation to $T$. Note that $T^{(\ell)}$ is a union of squares of side length $m^{-\ell}$, so it is geometrically a very simple object. It is easy to see that $\lim _{\ell \rightarrow \infty} T^{(\ell)}=T$ in the Hausdorff metric, but it is not true that $b\left(T^{(\ell)}\right)$ converges to $b(T)$. What can go wrong is that a "bay" becomes an "inland sea" in the limit. In other words, portions of $b\left(T^{(\ell)}\right)$ may belong to the outer boundary thanks to a path to infinity that slips through a narrow opening, but in the limit this narrow opening gets choked off.

For this reason we also consider another approximation to $T$. Let $\widetilde{S}$ denote the union of all squares $(S+i)$ for $i \in \mathbb{Z}^{2}$ that intersect $T$, so $T \subseteq \widetilde{S}$. It is not difficult to compute $\widetilde{S}$ exactly, or at least get an outer approximation (which will do as well). Let

$$
\widetilde{T}^{(\ell)}=\bigcup_{d \in \mathcal{D}_{\ell}}\left(m^{-\ell} \widetilde{S}+d\right) .
$$

Note that $T \subseteq \widetilde{T}^{(\ell)}$, so we are approximating $T$ from the outside, and we still have $T=\lim _{\ell \rightarrow \infty} \widetilde{T}^{(\ell)}$. In this case we have

$$
b(T)=\lim _{\ell \rightarrow \infty} b\left(\widetilde{T}^{(\ell)}\right),
$$

and points in $B(T) \backslash b(T)$ will never be approximated by points in $b\left(\widetilde{T}^{(\ell)}\right)$. Of course, we don't know a priori how large we have to take $\ell$ before $b\left(\widetilde{T}^{(\ell)}\right)$ gives a reasonable approximation of $b(T)$. As a practical matter we will consider $b\left(\widetilde{T}^{(\ell)}\right)$ to be a good approximation when it is indistinguishable from $b\left(\widetilde{T}^{(\ell-1)}\right)$. Because $\widetilde{T}^{(\ell)}$ is just a union of squares, it is easy to give an algorithm to find $b\left(\widetilde{T}^{(\ell)}\right)$ simply by "tracing" around $\widetilde{T}^{(\ell)}$ counterclockwise.

In order to describe $b(T)$ as the invariant set of an IFS, we need to decompose it into a finite union of basic pieces. There are several ways to approach this task. We will use a method based on "configurations." Let $A \subseteq \mathbb{Z}^{2}$ be any finite set containing 0 . The associated configuration $\mathcal{C}_{A}$ is defined to be

$$
\mathcal{C}_{A}=\bigcup_{i \in A} T_{i}
$$

(note that $T \subseteq \mathcal{C}_{A}$ ). The associated subset $b(T)_{A}$ of $b(T)$ is defined to be

$$
b(T)_{A}=b(T) \cap b\left(\mathcal{C}_{A}\right)=T \cap b\left(\mathcal{C}_{A}\right) .
$$


Informally, $b(T)_{A}$ is the part of $b(T)$ that is not "blocked off" by the other tiles in the configuration. We say two configurations $\mathcal{C}_{A}$ and $\mathcal{C}_{A^{\prime}}$ are equivalent if $b(T)_{A}=$ $b(T)_{A^{\prime}}$. In general, we have not been able to find an algorithm to determine whether or not two configurations are equivalent. In the examples we have looked at, it is easy to decide this, although it would be extremely tedious to prove equivalences. So this is the part of our work that requires "human intervention."

A tile will be said to satisfy the finiteness condition if there is only a finite set of equivalence classes of configurations. All the examples we have looked at satisfy this condition, and it is difficult to imagine a tile that does not. For the remainder of this section, we will discuss only tiles that satisfy it. Let $\left\{b(T)_{A_{k}}\right\}_{k=1, \ldots, N}$ denote a complete set of configuration pieces of $b(T)$. It is then easy to describe a graph-directed IFS that has this as an invariant set. The idea is to use Equation (1-3) to replace each tile in $\mathcal{C}_{A_{k}}$ by a union of tiles of level 1 . In particular,

$$
T=\bigcup_{d \in \mathcal{D}} m^{-1} T_{d}
$$

so that

$$
b(T)_{A_{k}}=\bigcup_{d \in \mathcal{D}} b(T)_{A_{k}} \cap m^{-1} T_{d} .
$$

Of course we can write $m^{-1} T_{d}=F_{d}(T)$ for $F_{d}(x)=$ $m^{-1}(x+d)$. Then $b(T)_{A_{k}} \cap m^{-1} T_{d}=F_{d}(T \cap$ $\left.F_{d}^{-1}\left(b(T)_{A_{k}}\right)\right)$. The claim is that $T \cap F_{d}^{-1}\left(b(T)_{A_{k}}\right)$ is just $b(T)_{A_{j}}$ for some $j$ (depending on $k$ and $d$ ). Indeed $T \subseteq F_{d}^{-1} T$ so $T \cap F_{d}^{-1}\left(b(T)_{A_{k}}\right)=T \cap b\left(F_{d}^{-1} \mathcal{C}_{A_{k}}\right)$ and $F_{d}^{-1} \mathcal{C}_{A_{k}}$ is another configuration that must be equivalent to some $\mathcal{C}_{A_{j}}$. Thus we have

$$
b(T)_{A_{k}}=\bigcup_{d \in \mathcal{D}} F_{d}\left(b(T)_{A_{j(k, d)}}\right)
$$

as desired. Since many of the pieces $b(T)_{A_{j(k, d)}}$ appearing in Equation (1-13) may be the empty set, we may safely omit them from the union. (Also, Equation (1-13) may contain some redundancy which may be pared away if desired.) It is also easy to see that the open set condition holds, as we may take $\operatorname{int}(T)$ as the open set associated with each piece, so in place of Equation (1-13) we have

$$
\bigcup_{d \in \mathcal{D}} F_{d}(\operatorname{int}(T)) \subseteq \operatorname{int}(T),
$$

and the union is disjoint. Since each mapping $F_{d}$ in Equation $(1-13)$ has the same contraction ratio $m^{-1}$ we may also use Equation (1-13) to compute the dimension of $b(T)$. We form the $N \times N$ matrix $M$ by setting

$$
M_{k j}=\#\{d: j(k, d)=j\} .
$$

Then

$$
\operatorname{dim} b(T)=\log \text { spec.rad. }(M) / \log m .
$$

Moreover, if $M$ is irreducible (it may require pruning, such as the removal of the empty set, to achieve this) then the pieces $b(T)_{A_{k}}$ all have finite positive Hausdorff measure, and the eigenvector associated to the largest eigenvalue of $M$ gives the relative measures of the pieces.

The pieces $b(T)_{A_{k}}$ may not be the most natural from a geometric point of view. In some examples we have examined they turn out to be disconnected (with infinitely many components), whereas it is possible to find (by careful inspection) a decomposition of $b(T)$ into connected pieces governed by a different graph directed IFS.

In Section 2, we prove that the boundary of any component of the interior decomposes into pieces similar to subsets of the outer boundary. In Section 3, we present several interesting examples of tiles satisfying the finiteness condition and show how to carry out the configuration method explicitly.

Theorem 2.1 and the configuration algorithm extend to more general self-similar tilings that involve rotations as well as homotheties in describing the self-similar identity. One example, the Lévy dragon [Lévy 38] (translated into English in [Edgar 93]), was in fact the motivating example for this work. In [Bailey et al. 02] it was noted that the boundaries of components of the interior have dimension one, despite the fact that the whole boundary has dimension $\approx 1.934007$, and in fact that component boundaries are infinite polygons. It is clear from inspection that the outer boundary is just an infinite polygon.

We note some interesting questions for future research:

(1) Does the finiteness condition always hold?

(2) What is the nature of the subsets of $b(T)$ in Theorem 2.1 ?

(3) Is there a computable upper bound for the number of similarity equivalence classes of components of the interior?

(4) Is there a construction of examples where the dimension of $b(T)$ gets arbitrarily close to 2 ? In [Kenyon et al. 99] examples are given where $\operatorname{dim} B(T)$ approaches 2 , but these examples will not suffice.

(5) Is it always true that each point of $b(T)$ may be connected by a path to infinity in the complement of $T$ ? This appears to be true for all our examples. 
More detailed results and code for the programs we used may be found on the website www.math.cornell.edu/ sld32/Tiles.html.

\section{BOUNDARIES OF COMPONENTS OF THE INTERIOR}

Let $C$ denote any component of int $(T)$.

Theorem 2.1. $B(C)=\bigcup_{i=1}^{N_{0}} B_{i}$ where each $B_{i}$ is similar to a subset of $b(T)$.

Proof: Consider the tiling (1-5) of level $\ell$. Each point of $B(C)$ must belong to at least one tile $m^{-\ell} T+i$ for $i$ not in $\mathcal{D}_{\ell}$. By compactness, the number of such tiles is finite. Set $B_{i}=B(C) \cap\left(m^{-\ell} T+i\right)$ (slight abuse of notation). Clearly each point of $B_{i}$ belongs to $B\left(m^{-\ell} T+i\right)$. To complete the proof we need to show that, for $\ell$ large enough, $B_{i} \subseteq b\left(m^{-\ell} T+i\right)$. Since $i$ is not in $\mathcal{D}_{\ell}, C$ is disjoint from $m^{-\ell} T+i$. Since $C$ is connected it belongs to one component of the complement of $m^{-\ell} T+i$. Which component? If we choose $\ell$ large enough, then $m^{-\ell} T+i$ will be small relative to $C$, so the answer has to be the unbounded component. For a more quantitative statement, let $A$ be an upper bound for the areas of the bounded components of the complement of $T$. Then $m^{-\ell} A<\operatorname{area}(C)$ implies that $C$ is not in any bounded component of the complement of $m^{-\ell} T+i$. This shows $B_{i} \subseteq b\left(m^{-\ell} T+i\right)$.

\section{EXAMPLES}

\subsection{The "Pinwheel"}

The "pinwheel" is generated by nine similarities with contraction ratios $1 / 3$. Figure 1 shows $T^{(1)}$, which consists of nine images of the unit square. This may be described succinctly as reflecting out the four corner subsquares, one in each edge direction in a rotation invariant pattern (there are two possible chiralities). Figure 2 shows $T^{(2)}$ and Figure 3 shows $T^{(5)}$. Because $T^{(1)}$ is connected and contains all four corners of the square, it follows by induction that each $T^{(m)}$ is connected, hence $T$ is connected. It is easy to see that the convex hull of $T$ is a square of side length $\sqrt{\frac{10}{4}}$, so the area of $T$ is at most 2.5. However, routine estimates show that the area is less than 2 , hence the area must equal 1 and $T$ tiles with $\mathbb{Z}^{2}[\mathrm{~B}]$. It is natural to conjecture from Figure 3 that all components of the interior of $T$ are similar to the single shape, shown in Figure 4.

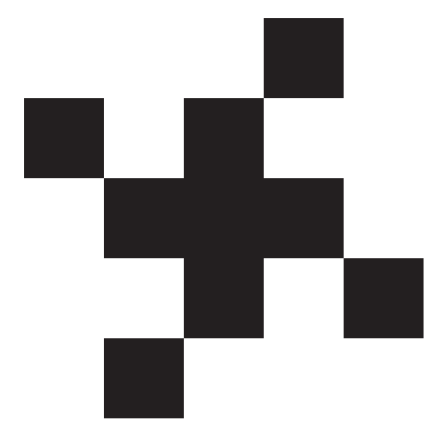

FIGURE 1. "Pinwheel" with $T^{(1)}$.

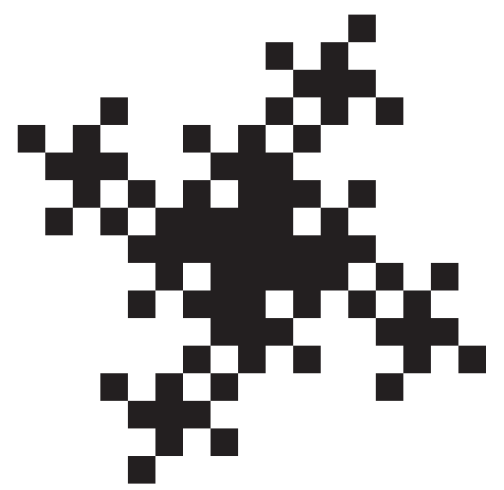

FIGURE 2. "Pinwheel" with $T^{(2)}$.

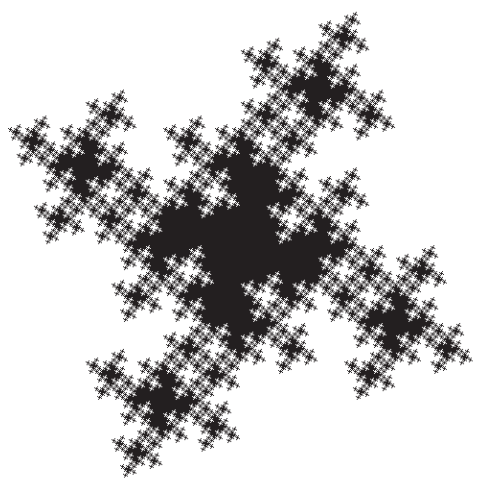

FIGURE 3. "Pinwheel" with $T^{(5)}$.

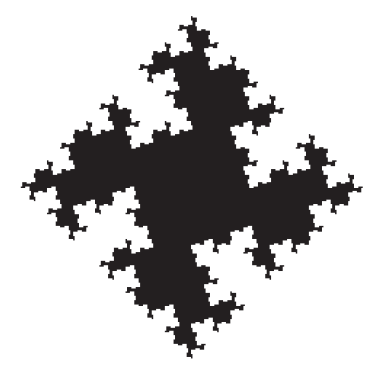

FIGURE 4. The components of the interior of $T$ are similar to this shape. 


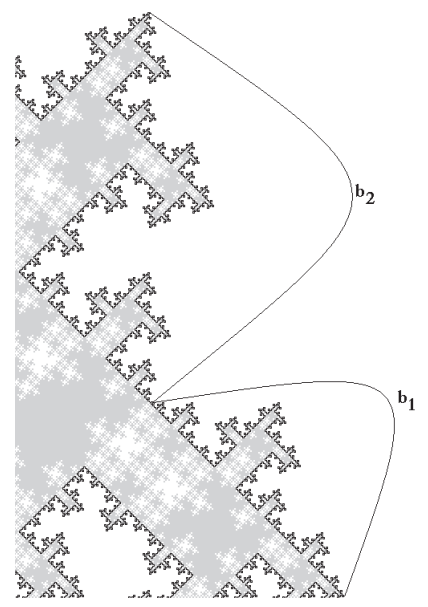

FIGURE 5. Decomposition of a side of $b$ into $b_{1}$ and $b_{2}$.

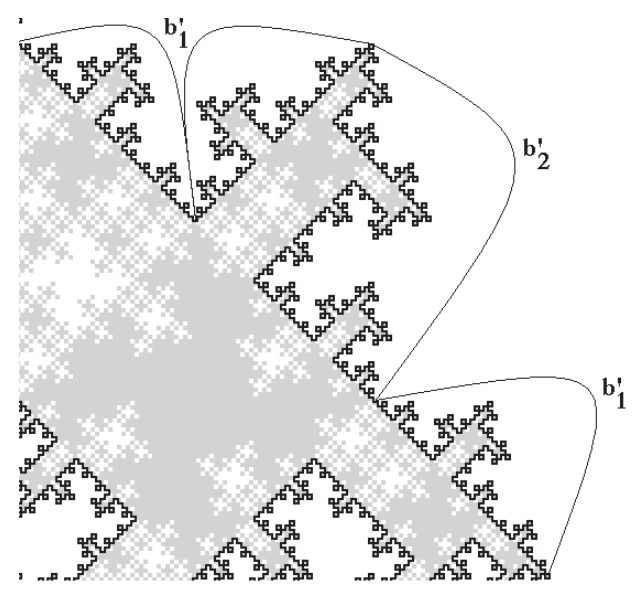

(a)

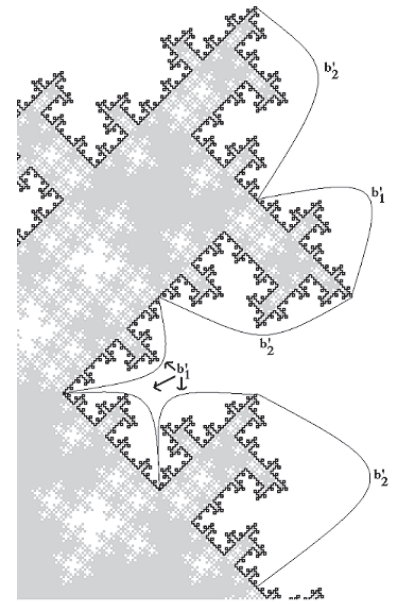

(b)

FIGURE 6. (a) Decomposition of $b_{1}$. (b) Decomposition of $b_{2}$. Both according to Equation (3-1).

To describe the outer boundary of $T$ we will exploit the rotational symmetry. We will need to use just two pieces, denoted $b_{1}$ and $b_{2}$. For $b_{1}$ we take $b(T)_{A}$ for the configuration shown in Figure 5, and we will identify the other three rotations of $b_{1}$ with $b_{1}$. The complement of the four copies of $b_{1}$ in $b$ clearly breaks into four isometric pieces, which we will call $b_{2}$. Note that if we think of $b(T)$ as being made up of four sides, then each side is made up of $b_{1} \cup b_{2}$, with the intersection $b_{1} \cap b_{2}$ consisting of a single point. Both $b_{1}$ and $b_{2}$ are connected. (Neither the sides nor the $b_{2}$ pieces can be described as $b(T)_{A}$ for any configuration.) If we denote by $b_{1}^{\prime}$ and $b_{2}^{\prime}$ images of $b_{1}$ and $b_{2}$ on level 1 , then

$$
\left\{\begin{array}{l}
b_{1}=b_{1}^{\prime} \cup b_{1}^{\prime} \cup b_{2}^{\prime} \cup b_{1}^{\prime} \\
b_{2}=b_{2}^{\prime} \cup b_{1}^{\prime} \cup b_{2}^{\prime} \cup b_{1}^{\prime} \cup b_{1}^{\prime} \cup b_{1}^{\prime} \cup b_{2}^{\prime}
\end{array}\right.
$$

is a decomposition analogous to Equation (1-13). Figure 6 illustrates this decomposition. The matrix (1-14) is then

$$
\left(\begin{array}{ll}
3 & 1 \\
4 & 3
\end{array}\right)
$$

with spectral radius 5 and associated eigenvalue $\left(\begin{array}{l}1 \\ 2\end{array}\right)$. Thus $\operatorname{dim} b(T)=\log 5 / \log 3$ by Equation (1-15), and the piece $b_{2}$ has twice the measure of $b_{1}$. The boundary of the component of the interior shown in Figure 4 decomposes into four copies of $b_{1}^{\prime}$ and four copies of $b_{2}^{\prime}$, as the reader may verify.

\subsection{The "Badge and Hydrant"}

The "badge and hydrant" tile is generated by 16 similarities with contraction ratio $1 / 4$. Figure 7 shows $T^{(1)}$, 

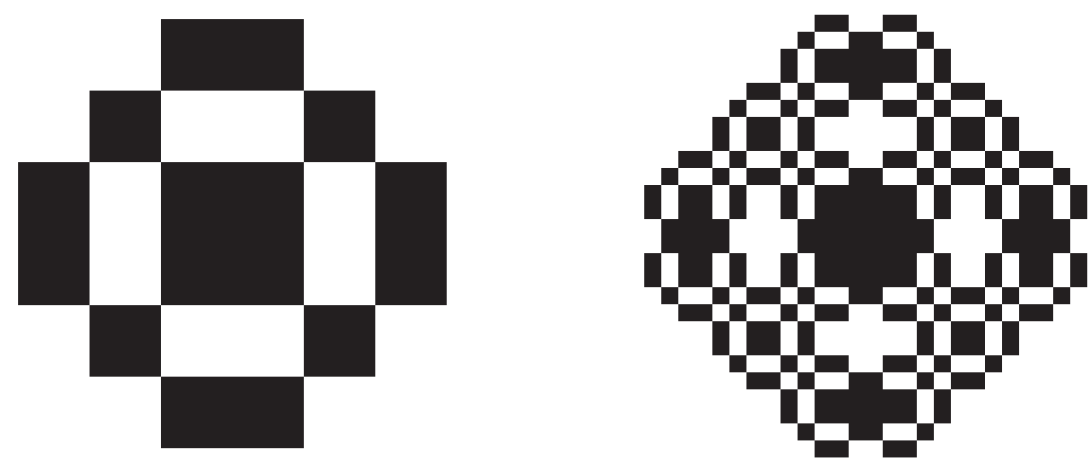

FIGURE 7. "Badge and Hydrant" with $T^{(1)}$.

FIGURE 8. "Badge and Hydrant" with $T^{(2)}$.
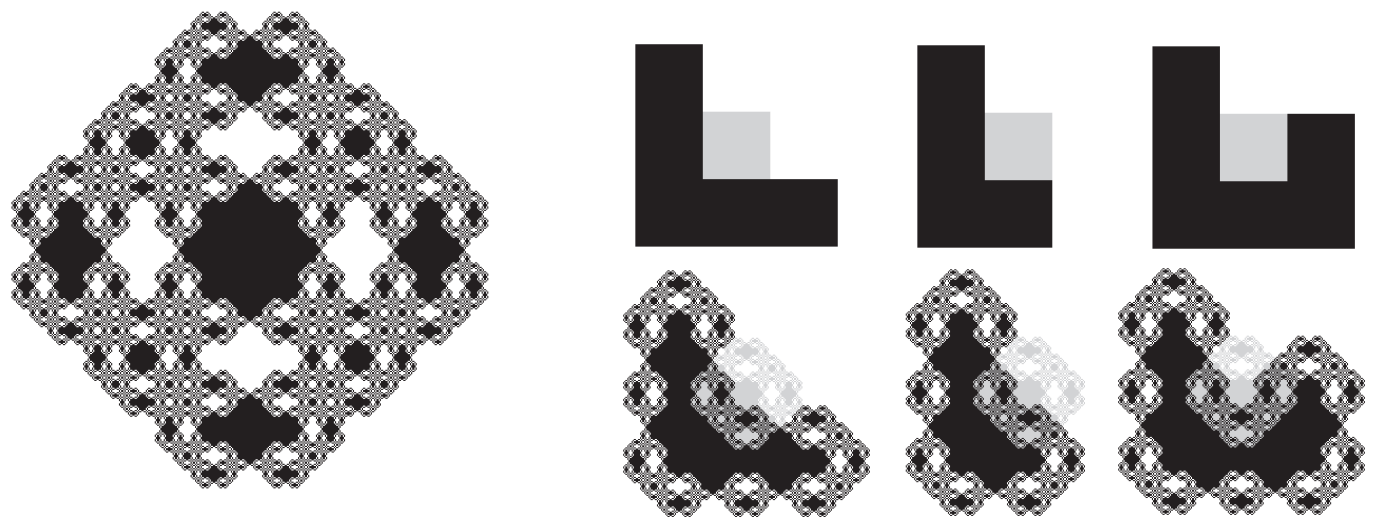

FIGURE 9. "Badge and Hydrant" with $T^{(5)}$.

FIGURE 10. $b_{1}, b_{2}$, and $b_{3}$ identified with $b(T)_{A}$.
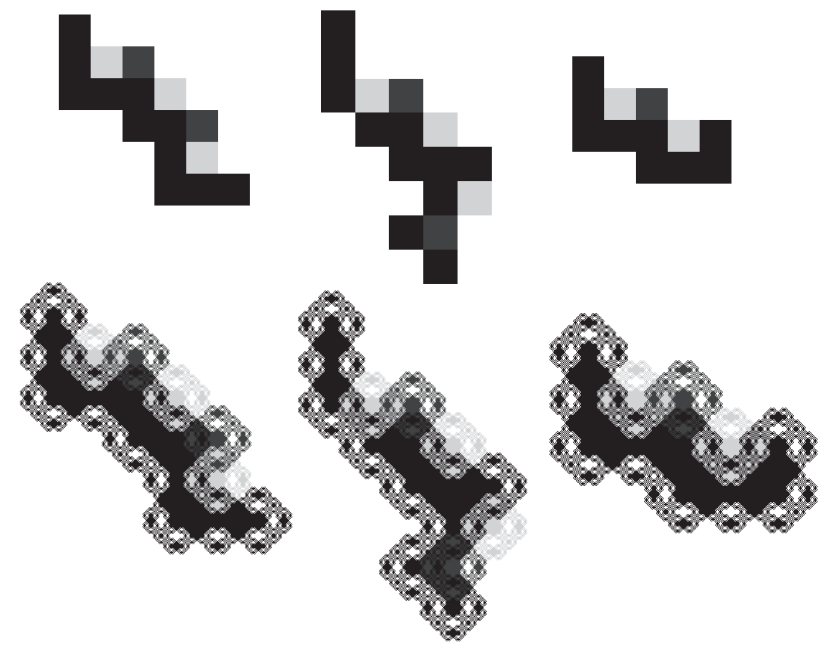

FIGURE 11. Schematic illustration of the decomposition of $b_{1}, b_{2}$, and $b_{3}$ according to Equation (3-2). 


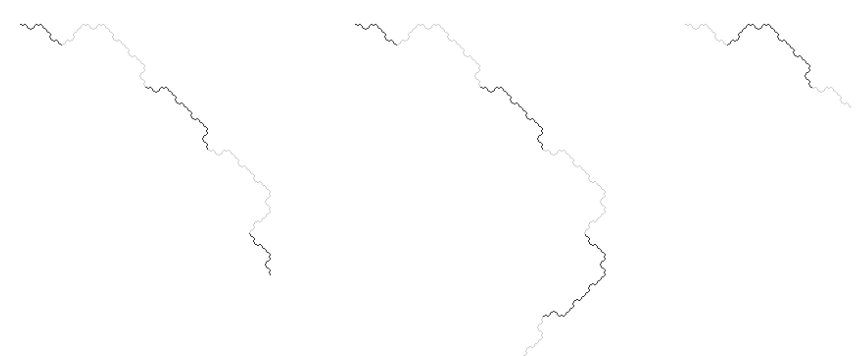

FIGURE 12. Actual boundary sets and their decomposition.

which consists of 16 images of the unit square. The corner and interior subsquares are retained, while the other edge subsquares are reflected out across the edge. Figure 8 shows $T^{(2)}$ and Figure 9 shows $T^{(5)}$. By the same reasoning as in Section 3.1, we may conclude that $T$ is connected and tiles with $\mathbb{Z}^{2}$. In Figure 9 we see two similarity types of interior components, the "badge" at the center and the "hydrants" (appearing in two distinct orientations).

We consider three pieces of the outer boundary $b_{1}$, $b_{2}, b_{3}$, each identified with $b(T)_{A}$ for the configurations shown in Figure 10. We identify rotated copies of the $b_{j}$. The entire outer boundary decomposes into four copies of $b_{1}^{\prime}$ and eight copies of $b_{2}^{\prime}$ consisting of the intersection of $b(T)$ with the 12 level 1 tiles $F_{j} T$ lying along the outer edge (all except the four interior tiles in Figure 7). Figure 11 illustrates schematically the decomposition

$$
\left\{\begin{array}{l}
b_{1}=b_{3}^{\prime} \cup b_{2}^{\prime} \cup b_{1}^{\prime} \cup b_{2}^{\prime} \cup b_{3}^{\prime} \\
b_{2}=b_{3}^{\prime} \cup b_{2}^{\prime} \cup b_{1}^{\prime} \cup b_{2}^{\prime} \cup b_{2}^{\prime} \cup b_{3}^{\prime} \\
b_{3}=b_{3}^{\prime} \cup b_{2}^{\prime} \cup b_{3}^{\prime} .
\end{array}\right.
$$

The actual sets and their decompositions are shown in Figure 12. Here the matrix (1-14) is

$$
\left(\begin{array}{lll}
1 & 2 & 2 \\
1 & 3 & 2 \\
0 & 1 & 2
\end{array}\right)
$$

with spectral radius $\frac{5+\sqrt{17}}{2}$, so the dimension is $\log \left(\frac{5+\sqrt{17}}{2}\right) / \log 4 \approx 1.0947625$.

The boundary of the badge component is made up of eight copies of $b_{3}^{\prime}$, each one corresponding to one of the eight level one tiles in the complement of $T$ (clearly visible in Figure 7). To see the boundary of the hydrant schematically we have to look at level two (Figure 8). There we see four copies of $b_{2}^{\prime \prime}$ and 12 copies of $b_{3}^{\prime \prime}$. We could also consolidate using Equation (3-2) to have four copies of $b_{3}^{\prime}$ and four copies of $b_{3}^{\prime \prime}$.

\subsection{The "Bug"}

The "bug" tile is generated by nine similarities with contraction ratio $\frac{1}{3}$. Figures $13-15$ show $T^{(1)}, T^{(2)}$, and $T^{(5)}$. In Figure 16 we show the outer boundary extracted from $\widetilde{T}^{(5)}$. This gives a much clearer notion of what the outer boundary actually looks like. This tile has only one symmetry, a reflection in the vertical axis. It appears that there are three distinct shapes among the components of the interior, one of which appears in two chiralities. These are shown in Figure 17.

To decompose the outer boundary into pieces $b(T)_{A}$ associated with different configurations $A$, we were initially led to a set of 19 configurations. We then were able to consolidate the list to 11 pieces, shown in Figure 18 together with the associated configuration and the decomposition on the next level. Note that the decomposition of piece 11 includes two copies of piece 7 contracted twice. To do the dimension computation we add piece 12 which is just $7^{\prime}$, and we obtain the $12 \times 12$ matrix (1-14) to be

$\begin{array}{llllllllllll}1 & 1 & 1 & 0 & 0 & 0 & 0 & 2 & 0 & 1 & 2 & 4 \\ 1 & 0 & 1 & 1 & 0 & 0 & 0 & 0 & 0 & 0 & 0 & 0 \\ 1 & 0 & 1 & 1 & 0 & 0 & 0 & 0 & 0 & 1 & 0 & 0 \\ 0 & 0 & 0 & 1 & 0 & 1 & 0 & 0 & 0 & 0 & 0 & 0 \\ 1 & 1 & 1 & 1 & 0 & 0 & 0 & 2 & 2 & 1 & 0 & 0 \\ 1 & 1 & 1 & 2 & 0 & 0 & 0 & 1 & 2 & 0 & 0 & 0 \\ 0 & 0 & 0 & 0 & 0 & 0 & 1 & 2 & 0 & 0 & 2 & 2 \\ 1 & 0 & 1 & 0 & 0 & 0 & 0 & 1 & 0 & 1 & 0 & 0 \\ 0 & 0 & 0 & 0 & 0 & 0 & 0 & 2 & 3 & 0 & 0 & 0 \\ 0 & 0 & 0 & 0 & 1 & 0 & 0 & 0 & 1 & 0 & 0 & 0 \\ 0 & 0 & 0 & 0 & 0 & 0 & 0 & 1 & 0 & 0 & 2 & 2 \\ 0 & 0 & 0 & 0 & 0 & 0 & 1 & 0 & 0 & 0 & 0 & 0\end{array}$

The spectral radius is $\approx 4.5007735787273$ so the dimension of the outer boundary is $\approx 1.3692267089143$.

We note that the pieces in the decomposition are not connected. It is possible to obtain a decomposition into connected pieces by ad hoc means, but the pieces are not associated with configurations. For example, a pair of pieces of type 7 gives a connected set (see the decomposition of piece 11 in Figure 18), but this new set is is not given by any configuration. 


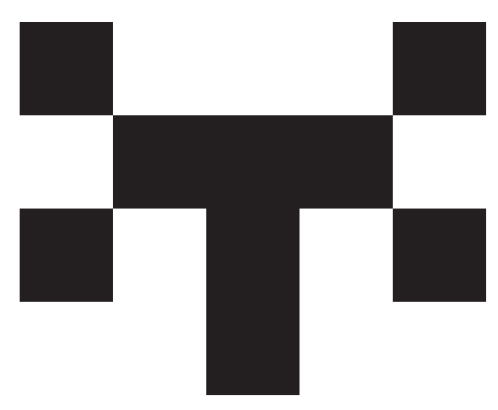

FIGURE 13. The "bug" with $T^{(1)}$.

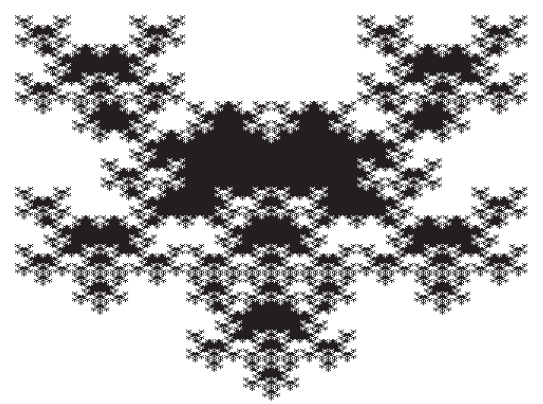

FIGURE 15. The "bug" with $T^{(5)}$.

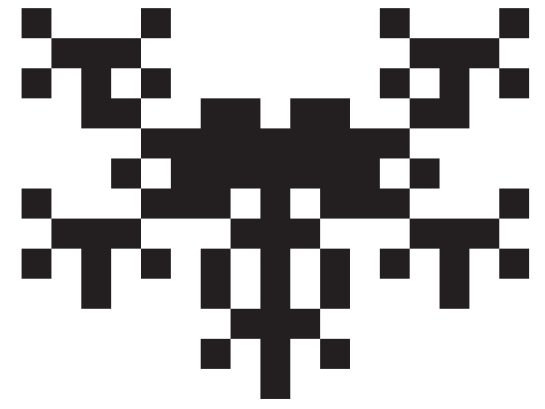

FIGURE 14. The "bug" with $T^{(2)}$.

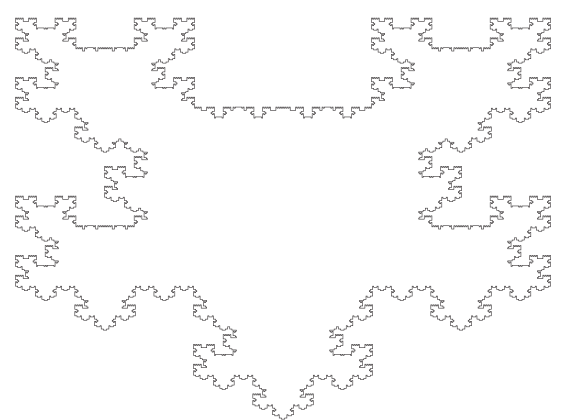

FIGURE 16. The outer boundary of the "bug" extracted from $\tilde{T}^{(5)}$.
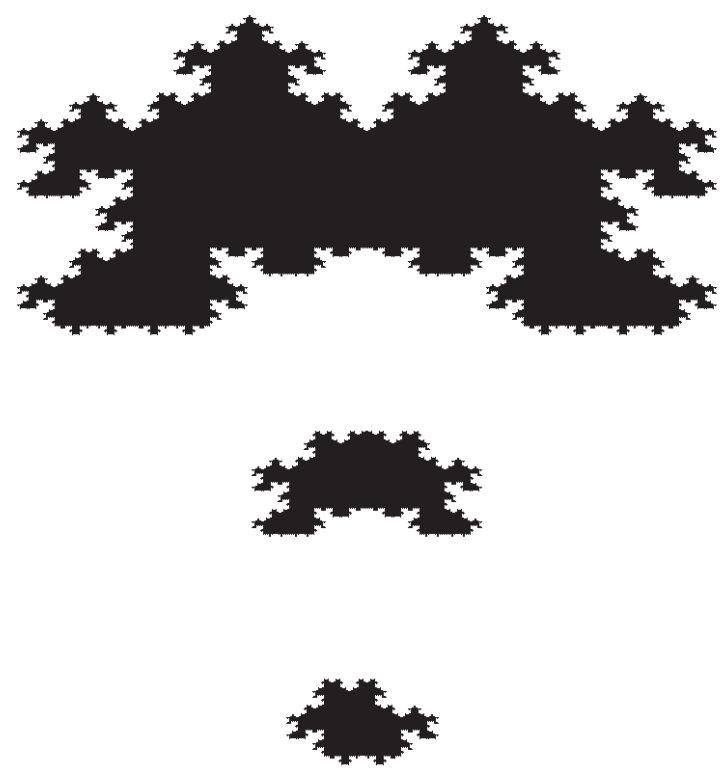

FIGURE 17. Shapes among the components of the interior of the "bug." 

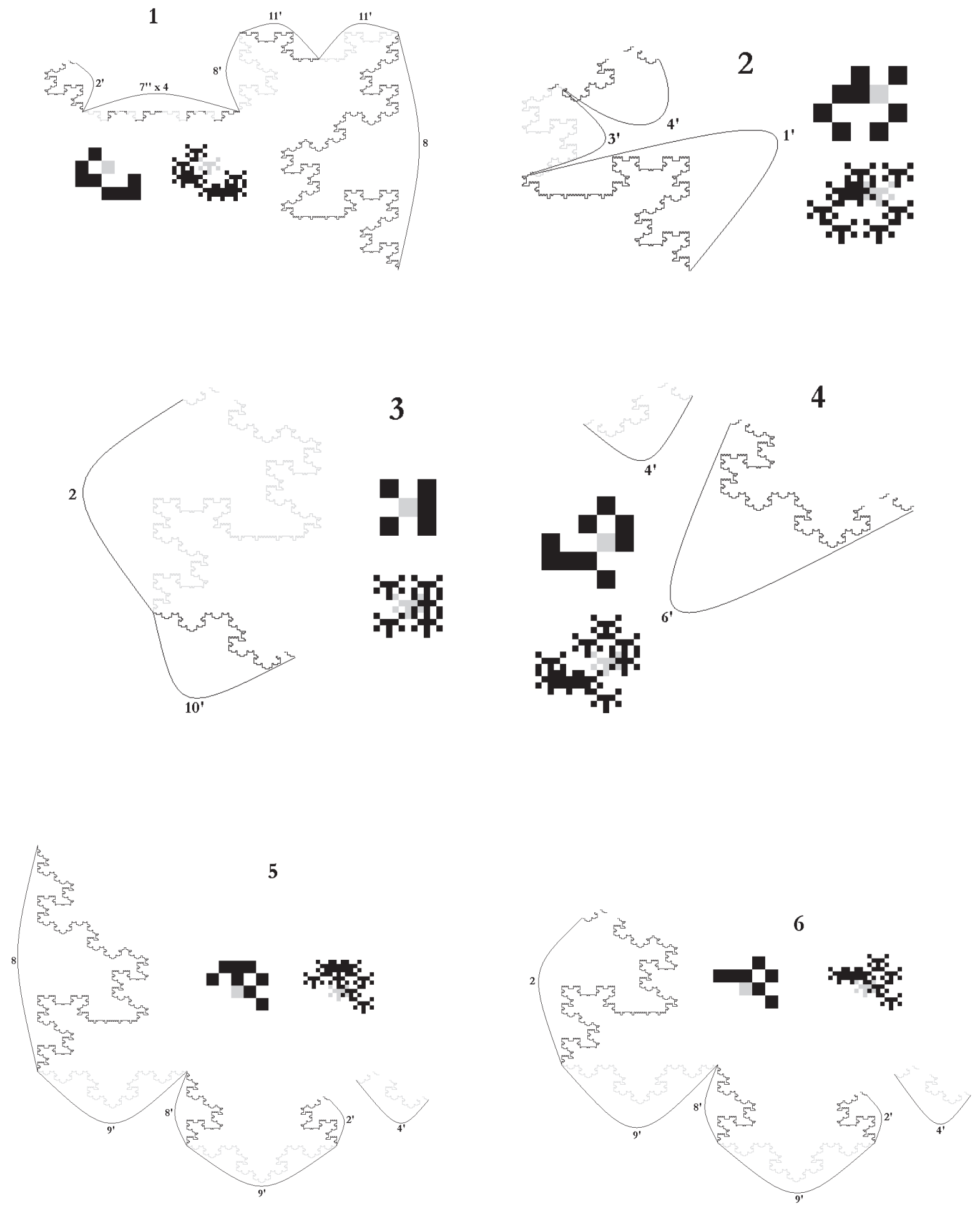

FIGURE 18. Decomposition of the "bug." 

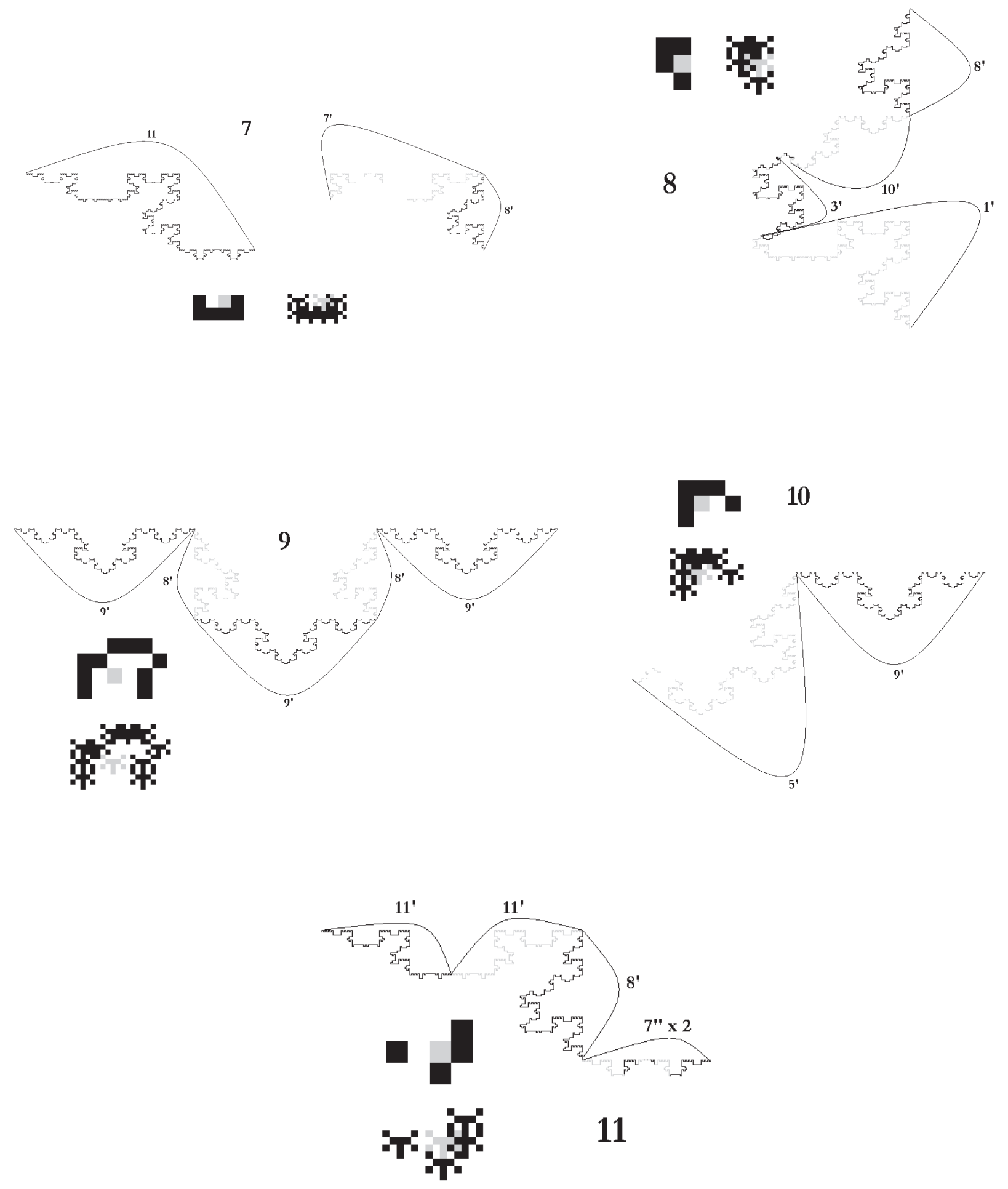

FIGURE 18. (continued.) 


\section{ACKNOWLEDGMENTS}

We are grateful to Matthew Maloney who helped with the computer experimentation. We are grateful to one of the referees for pointing out some weaknesses in the original version of this paper, and for suggesting problem 5. The first author's research was supported by a Cornell Presidential Research Scholarship. The second and third author thank the Cornell Mathematics Department for hospitality when this research was carried out while on professional leave from the University of Akron. The fourth author's research was supported in part by the National Science Foundation grant \#DMS-0140194.

\section{REFERENCES}

[Bailey et al. 02] S. Bailey, T. Kim, and R. S. Strichartz. "Inside the Lévy Dragon." Amer. Math. Monthly 109 (2002), 689-703.

[Bandt 91] C. Bandt. "Self-Similar Sets 5. Integer Matrices and Fractal Tilings of $\mathbb{R}^{n}$." Proc. Amer. Math. Soc. 112 (1991), 549-562.

[Bandt and Gelbrich 94] C. Bandt and G. Gelbrich. "Classification of Self-Affine Lattice Tilings." J. London Math. Soc. (2) 50 (1994), 581-593.

[Bandt and Wang 01] C. Bandt and Y. Wang. "Disk-Like Self-Affine Tiles in $\mathbb{R}^{2}$." Discrete Comput. Geom. 26:4 (2001), 591-601.

[Dubuc and Li 02] S. Dubuc and J. Li. "Le pavage du plan par la courbe de Lévy." Ann. Sci. Math. Québec 26 (2002), 147-159.

[Duvall and Keesling 97] P. Duvall and J. Keesling. "The Hausdorff Dimension of the Boundary of the Lévy Dragon." Int. J. Math. and Math. Sci. 20 (1997), 627632 .
[Edgar 93] G. A. Edgar. Classics on Fractals. Reading, MA: Addison-Wesley, 1993.

[Kenyon et al. 99] R. Kenyon, J. Li, R. S. Strichartz, and Y. Wang. "Geometry of Self-Affine Tiles II." Indiana Univ. Math. J. 48 (1999), 24-42.

[Kirat and Lau 00] I. Kirat and K. -S. Lau. "On the Connectedness of Self-Affine Tiles." J. London Math. Soc. (2) 62 (2000), 291-304.

[Kirat and Lau 02] I. Kirat and K. -S. Lau. "Classification of Integral Expanding Matrices and Self-Affine Tiles." Discrete Comput. Geom. 28 (2002), 49-73.

[Kirat et al. 04] I. Kirat, K. -S. Lau, and H. Rao. "Expanding Polynomials and Connectedness of Self-Affine Tiles." Discrete Comput. Geom. 31 (2004), 275-286.

[Lévy 38] P. Lévy. "Les courbes planes ou gauches et les surfaces composée de parties semblables au tout." J. d'Ecole Polytechnique (1938), 227-247, 249-291.

[Mauldin and Williams 88] R. D. Mauldin and S. C. Williams. "Hausdorff Dimension in Graph Directed Constructions." Trans. Amer. Math. Soc. 309 (1988), 811829.

[Ngai and Nguyen 03] S. -M. Ngai and N. Nguyen. "The Heighway Dragon Revisited." Discrete Comput. Geom. 29 (2003), 603-623.

[Ngai and Tang 05] S. -M. Ngai and T. -M. Tang. "Topology of Connected Self-Similar Tiles in the Plane with Disconnected Interiors." Topology Appl. 150 (2005), 139-155.

[Ngai et al. 00] S. -M. Ngai, V. Sirvent, J. J. P. Veerman, and Y. Wang. "On 2-Reptiles in the Plane." Geom. Dedicata 82 (2000), 325-344.

[Strichartz and Wang 99] R. S. Strichartz and Y. Wang. Geometry of Self-Affine Tiles, I." Indiana Univ. Math. J. 48 (1999), 1-23.

Shawn Drenning, Mathematics Department, University of Chicago, Chicago, IL 60637

(shawn@math.uchicago.edu)

Judith Palagallo, Dept. of Theoretical \& Applied Mathematics, University of Akron, Akron, OH 44325-4002 (palagallo@uakron.edu)

Thomas Price, Dept. of Theoretical \& Applied Mathematics, University of Akron, Akron, OH 44325-4002 (teprice@uakron.edu)

Robert S. Strichartz, Mathematics Department, Malott Hall, Cornell University, Ithaca, NY 14853 (str@math.cornell.edu)

Received November 11, 2004; accepted February 15, 2005. 